\title{
Tumeur myofibroblastique inflammatoire de la vessie
}

\author{
Wafa Jomaa, MD; Sonia Ziadi, MD; Mounir Trimeche, MD; Faten Hammedi, MD; Nabil Ben Sorba, MD; \\ Moncef Mokni, MD; Sadok Korbi, MD
}

\section{Résumé}

La tumeur myofibroblastique inflammatoire est une lésion classée par l'OMS parmi les néoplasies intermédiaires. Elle peut siéger dans différents organes : les poumons, le pancréas, le mésentère et l'utérus. La localisation vésicale est rare.

Nous rapportons le cas d'un jeune homme de 38 ans qui a consulté pour hématurie. L'échographie a révélé la présence d'une structure tissulaire intravésicale de $3 \mathrm{~cm}$ de grand axe. L'examen anatomopathologique et l'étude immunohistochimique ont conclu à une tumeur myofibroblastique inflammatoire de la vessie. À partir de cette observation, nous présentons les diagnostics différentiels et les particularités anatomopathologiques de cette localisation rare.

Cite as: Can Urol Assoc J 2011;5(1):e4-e6; D0I:10.5489/cuaj.10008

\section{Abstract}

Myofibroblastic inflammatory tumor is considered as an intermediate neoplasm according to the WHO classification. It can occur in different organs: lung, pancreas, mesentery and uterus. The localization in the bladder is unusual. We report a case of a 38-year-old patient who presented with hematuria. Echography showed a $3 \mathrm{~cm}$ tissular structure of the bladder. Histological analysis and immunohistochemistry concluded to the diagnosis of myofibroblastic tumor of the bladder. The present work will give a general view of the myofibroblastic tumor, and will review its differential diagnosis.

\section{Introduction}

Les tumeurs myofibroblastiques inflammatoires (TMI), connues sous plusieurs noms (pseudotumeur inflammatoire, granulome plasmocytaire, tumeur fibromyxoïde pseudosarcomateuse), constituent un groupe de tumeurs mésenchymateuses survenant surtout chez le sujet jeune. Elles sont observées dans un grand nombre d'organes et en particulier, les poumons. Quelques cas ont été décrits au niveau de la vessie. De récentes données de génétique moléculaire ont éclairé la controverse portant sur la nature de cette prolifération. En effet, la mise en évidence d'une anomalie non aléatoire de la bande chromosomique 2 p23 conduisant à un remaniement génique intéressant le gène $A L K$ plaide en faveur d'une origine néoplasique plutôt que réactionnelle des TMI. Nous signalons un nouveau cas de TMI de la vessie chez un jeune homme. À partir de cette observation et d'une revue de la littérature, nous présentons les diagnostics différentiels et les particularités anatomopathologiques de cette localisation rare.

\section{Observation}

Un jeune homme de 38 ans, sans antécédents pathologiques particuliers, consultait pour une hématurie évoluant depuis 3 mois. L'examen somatique et le bilan biologique étaient sans particularité. L'échographie abdomino-pelvienne a révélé la présence d'une structure tissulaire intravésicale échogène pariétale latérosupérieure droite de $3 \mathrm{~cm}$ de diamètre. La cystoscopie a permis de visualiser une lésion solide, recouverte $d^{\prime}$ une muqueuse $d^{\prime}$ aspect normal, qui a été réséquée en totalité.

L'examen histologique a montré qu'il s'agissait d'une prolifération de cellules fusiformes, à noyau ovalaire parfois nucléolé et à cytoplasme éosinophile, et qui prenaient parfois un aspect de type ganglionnaire. Ces cellules s'agençaient en faisceaux courts enchevêtrés et disposés dans une substance de fond discrètement myxoïde. Les atypies cytonucléaires étaient discrètes et l'index mitotique était faible, soit 2 mitoses (normales) pour 10 GC. La prolifération cellulaire était mêlée à un infiltrat inflammatoire abondant plus accentué en périphérie et riche en lymphocytes, plasmocytes et polynucléaires éosinophiles. La tumeur n'infiltrait pas la musculeuse et ne comportait pas de nécrose. Le revêtement excréto-urinaire était par ailleurs normal.

L'étude immunohistochimique mettait en évidence une expression cytoplasmique intense et diffuse par l'anticorps anti-ALK1 (anaplastic lymphoma kinase) et I'anticorps antivimentine, et une expression focale par l'anticorps antidesmine, ainsi qu'un marquage nucléaire par l'anticorps anti-p53. Devant l'aspect histologique et le profil immunohistochimique, notamment la positivité cytoplasmique avec 


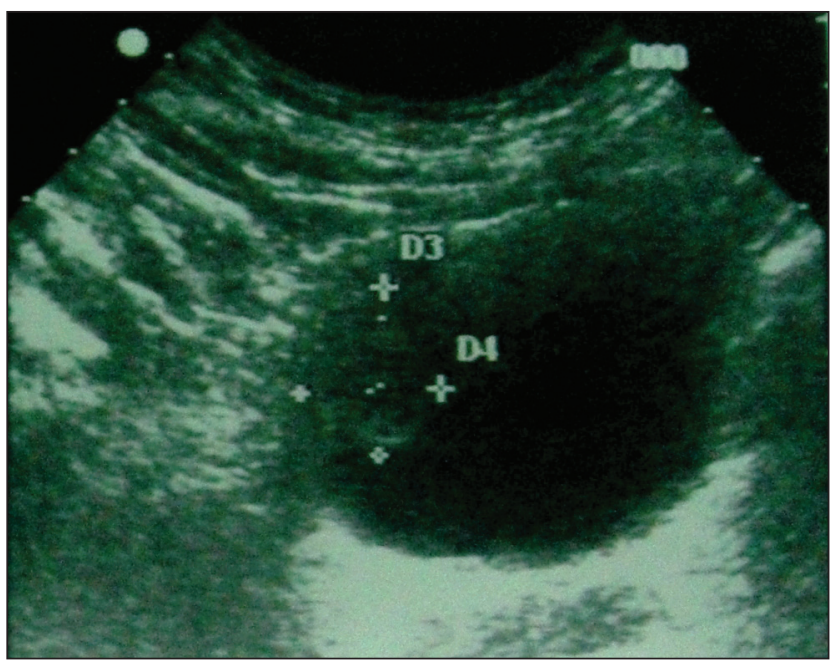

Fig. 1. Echographie de la vessie montrant une lésion intravésicale échogène pariétale latéro-supérieure droite de $3 \mathrm{~cm}$ de diamètre.

I'anticorps anti-ALK1, le diagnostic de tumeur myofibroblastique inflammatoire a été retenu.

On n'a noté aucune complication postopératoire et l'évolution était favorable, sans récidive locale ou à distance 6 mois plus tard.

\section{Discussion}

La TMI est une lésion classée par l'OMS parmi les néoplasies intermédiaires ${ }^{1}$. Elle peut siéger dans différents organes : les poumons, le pancréas, le mésentère et l'utérus. La localisation vésicale est rare ${ }^{2,3}$. C'est une lésion qui survient essentiellement chez l'adulte jeune mais qui peut s'observer chez l'enfant et le sujet âgé, avec une prédominance masculine ${ }^{3}$. L'hématurie est le signe révélateur le plus fréquent ${ }^{2,4}$ et constituait le motif de consultation de notre patient.

À l'imagerie, la TMI ne présente pas de spécificité. L'échographie et la TDM permettent de mettre en évidence une masse intravésicale souvent volumineuse et hétérogène pouvant comporter des calcifications ${ }^{2}$. La cystoscopie met en évidence une masse exophytique polypoïde ou nodulaire, parfois ulcérée, à base d'implantation large ${ }^{5,6}$.

Sur le plan histologique, il s'agit d'une prolifération de cellules fusiformes à cytoplasme éosinophile correspondant à des myofibroblastes. Les noyaux peuvent être de grande taille, parfois atypiques, comportant un nucléole proéminent. L'activité mitotique est variable. On peut parfois observer des myofibroblastes immatures ressemblant aux cellules ganglionnaires. Un infiltrat inflammatoire chronique lymphoplasmocytaire est noté dans les cas typiques, pouvant comporter des polynucléaires éosinophiles ou neutrophiles. Le stroma est lâche, œdémateux ou myxoïde et peut compter un nombre important de vaisseaux sanguins. Une infiltration de la musculeuse ou du tissu périvésical peut être observée 5 .

Sur le plan immunohistochimique, la TMI exprime la

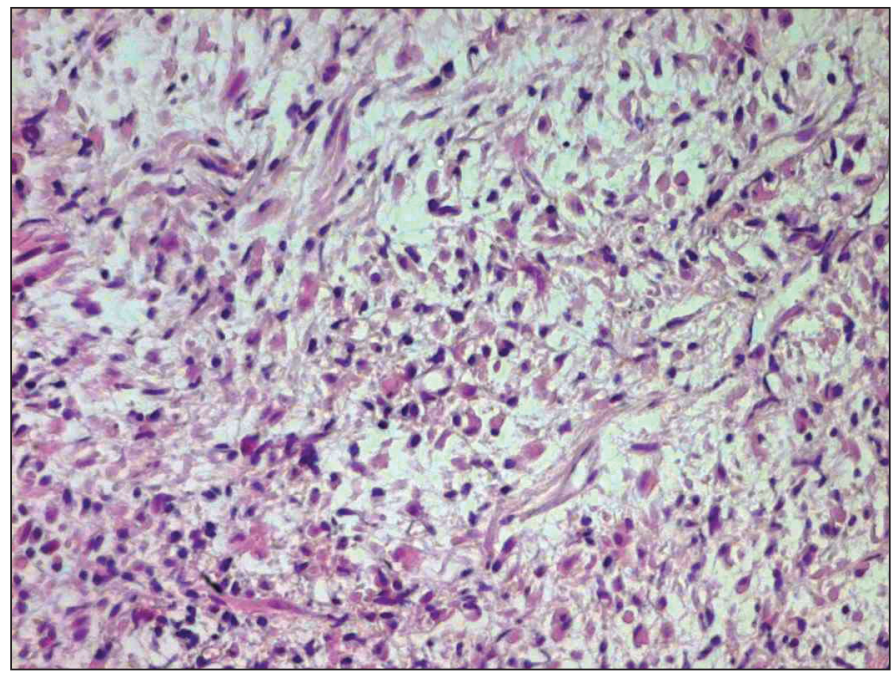

Fig. 2. Prolifération de faisceaux courts de cellules fusiformes disposées dans une substance de fond myxoïde $(\mathrm{HE} \times 200)$.

vimentine (95 à $100 \%$ ) et la desmine (5 à $80 \%$ ); ces deux marqueurs sont exprimés chez notre patient. Elle exprime également l'AML (48 à $100 \%$ ) et la kératine (10 à $89 \%$ ). Elle est négative pour les marqueurs du muscle squelettique, à savoir la myoglobine, la myogénine et la MyoD13,5.

L'ALK1 est exprimée dans 8 à $89 \%$ des TMI de la vessie. Notre patient a présenté un marquage cytoplasmique intense et diffus pour cette protéine.

Des études récentes de génétique moléculaire ont révélé une aberration chromosomique touchant le bras court du chromosome 2 au niveau de la région p21-p23. Il s'agit d'un réarrangement du gène codant I'ALK, consolidant le concept d'une origine néoplasique, plutôt qu'inflammatoire ou réactionnelle, des TMI. Plusieurs translocations ont été mises en évidence par la technique de FISH, la plus fréquente étant la $\mathrm{t}(1 ; 2)(\mathrm{q} 25 ; \mathrm{p} 23)^{3,5,7}$.

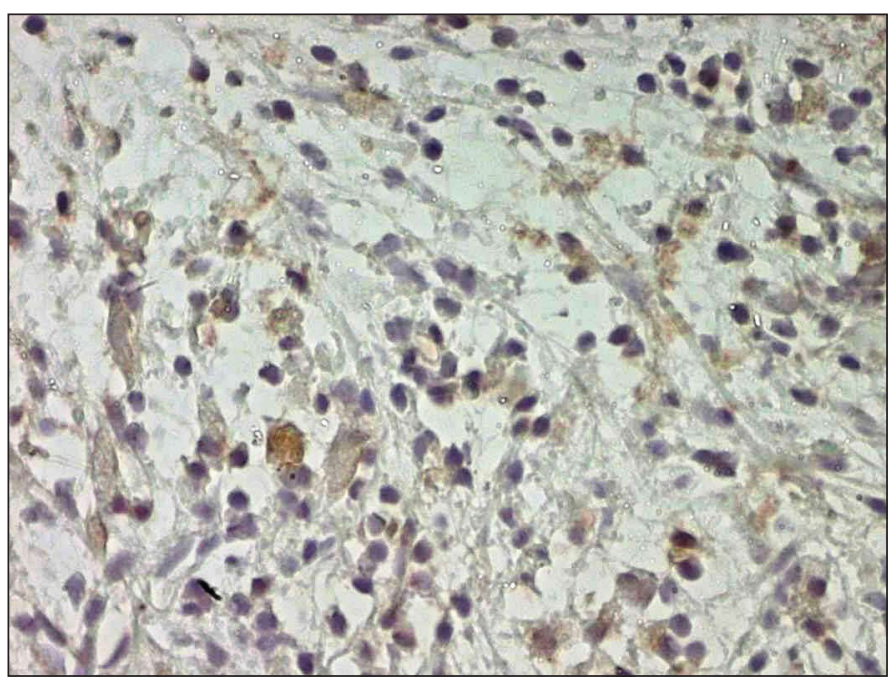

Fig. 3. Immunomarquage cytoplasmique intense et diffus par l'anticorps antiALK 1 (IHC x400) 
Jomaa et al.

La positivité de l'ALK serait prédictive d'un bon pronostic. En effet, deux études menées sur des séries de 61 et 15 cas de TMI ont mis en évidence l'absence de récurrence et de métastase à distance pour les cas $\mathrm{ALK}+{ }^{8,9}$.

Le diagnostic différentiel se pose essentiellement avec la prolifération myofibroblastique pseudosarcomateuse (PMP). Cette lésion qui présente des aspects morphologiques et immunohistochimiques similaires à la TMI diffère tant par son origine réactionnelle plutôt que tumorale que par son évolution bénigne et dépourvue de récidive ou de métastase à distance. Elle s'observe à tout âge, généralement suite à un geste endoscopique. Histologiquement, elle se caractérise par un phénomène de zones qui fait alterner des zones peu cellulaires à fond lâche et myxö̈de avec des zones plus cellulaires, souvent d'architecture fasciculée. Sur le plan immunohistochimique, elle exprime la cytokératine de façon intense; I'expression de l'ALK est moins fréquente. Sur le plan moléculaire, il n'existe pas de réarrangement génétique, contrairement à la $\mathrm{TM}^{3} \mathrm{3}^{30}$.

Deux tumeurs malignes doivent impérativement être écartées : le léiomyosarcome de type myxö̈de et le rhabdomyosarcome embryonnaire. Le léiomyosarcome comporte des atypies plus marquées et des mitoses plus nombreuses et il exprime l'AML de façon plus intense et plus diffuse. Le rhabdomyosarcome embryonnaire présente une cellularité élevée avec un nombre important de mitoses atypiques parfois accompagnées de striations cytoplasmiques et il est positif pour la myogénine et le myo-D1. Ces deux tumeurs, contrairement à la TMI, n'expriment pas I'ALK 5,11 .

La prise en charge thérapeutique consiste en une résection endoscopique complète. Un traitement intensif (cystectomie radicale, radiothérapie ou chimiothérapie) est inutile vu l'évolution bénigne dans la majorité des cas. Cependant, une surveillance étroite est recommandée afin de détecter une éventuelle récidive ${ }^{3}$. Notre patient a eu une résection endoscopique de la tumeur, jugée complète, avec une évolution favorable 6 mois plus tard.

\section{Conclusion}

La TMI est une lésion rare de la vessie située à mi-chemin entre les tumeurs bénignes et les tumeurs malignes. Elle doit être identifiée afin d'être traitée d'une façon convenable et d'éviter aux malades des thérapeutiques intensives inutiles.
Laboratoire d'anatomie et de cytologie pathologiques, CHU Farhat Hached, 4000 Sousse, Tunisie

Competing interests: None declared.

This paper has been peer-reviewed.

\section{Bibliographie :}

1. Fletcher CDM, Mertens F. World Health Organization classification of tumors: pathology and genetics of tumors of soft tissue and bone. Lyon (France): IARC Press; 2002.

2. Ali S, El Kettani N, Dafiri R. Tumeur myofibroblastique inflammatoire de la vessie. Fevillets de radiologie $2007,47, n^{\circ} 2,113-118$.

3. Cheng L, Foster SR, MacLennan GT, Lopez-Beltran A, Zhang S, Montironi R. Inflammatory myofibroblastic tumors of the genitourinary tract- single entity or continuum? I Urol 2008; 180:1235-40.

4. Gomez Garcia I , Molina Burgos R, Fernandez E, et al. Myofibroblastic tumor of bladder. Acta Urol Esp 2005:611-4

5. Lott S, Lopez-Beltran A, Montironi R, et al. Soft tissue tumors of the urinary bladder, part l: myofibroblastic proliferations, benign neoplasms, and tumors of uncertain malignant potential. Hum Pathol 2007:38:807-23

6. Lantz $A G$, Power NE, Gupta $R$, et al. Inflammatory pseudotumor: a rare cause of hematuria and shock. Urology 2007;70:372.e3-6.

7. Freeman A, Geddes N, Munson P, et al. Anaplastic lymphoma kinase (ALK 1) staining and molecular analysis in inflammatory myofibroblastic tumours of the bladder: a preliminary clinicopathological study of nine cases and review of the literature. Mod Pathol 2004;17:765-71.

8. Chan JK, Cheuk W, Shimizu M. Anaplastic lymphoma kinase expression in inflammatory pseudotumors. Am I Surg Pathol 2001;25:761-8.

9. Jiang YH, Cheng B, Ge MH, et al. Comparison of the clinical and immunohistochemical features, including anaplastic lymphoma kinase (ALK) and p53, in inflammatory myofibroblastic tumours. J Int Med Res 2009;37:867-77.

10. Nim L, et al. Prolifération myofibroblastique pseudosarcomateuse de la vessie. Prog Urol (2009), DOI : 10.1016/i.purol.2009.05.007.

11. Sukov WR, Cheville JC, Carlson AW, et al. Utility of ALK-1 protein expression and ALK rearrangements in distinguishing inflammatory myofibroblastic tumor from malignant spindle cell lesions of the urinary bladder. Mod Pathol 2007;20:592-603. Epub 2007 Mar 30.

Correspondence: Dr Wafa Jomaa, Laboratoire d'anatomie et de cytologie pathologiques, Hôpital Farhat Hached, 4000 Sousse, Tunisie ; télécopieur : 21673210355 ; wafaioma@@hotmail.fr 